\title{
Spectrum of Lyapunov exponents in non-smooth systems evaluated using orthogonal perturbation vectors
}

\author{
Marek Balcerzak ${ }^{1}$, Artur Dąbrowski ${ }^{1}$, Andrzej Stefański ${ }^{1 *}$, and Jerzy Wojewoda $^{1}$ \\ ${ }^{1}$ Division of Dynamics, Lodz University of Technology, Stefanowskiego 1/15, 90-924 Lodz, Poland
}

\begin{abstract}
This paper covers application of the novel method of Lyapunov exponents (LEs) spectrum estimation in non smooth mechanical systems. In the presented method, LEs are obtained from a Poincaré map. By analysing the map instead of the full trajectory, problems with transition of perturbations through discontinuities can be avoided. However, the explicit formula of the map is usually not known. Therefore, the Jacobi matrix of the map is estimated using small perturbations of the initial point. In such a manner, direct calculation of the Jacobi matrix can be avoided. The article provides a detailed description of the method accompanied by clear schemes. The algorithm of Jacobi matrix estimation is elaborated and an example is given. Efficiency of the method is confirmed by a numerical experiment. The mechanical oscillator with impact has been simulated. Bifurcation diagrams and Lyapunov exponents graphs have been generated. It has been shown that the method provides values of the whole Lyapunov exponents spectrum with high accuracy.
\end{abstract}

\section{Introduction}

Lyapunov exponents (LEs) are one of the most useful criteria for determining the stability of solutions of dynamical systems in local [1] and also global sense [2]. They are extremely useful instruments for identification of the motion character in these systems. Determination of these numbers is one of the fundamental tasks in nonlinear dynamics. Definition of LEs was established by V. I. Oseledec [3], in a form suited for the theory of dynamical systems. These numbers were named after A. M. Lyapunov, due to the fact that their values are qualitative and quantitative illustration of his criterion of dynamical systems stability [1]. LEs can be treated as the exponential measure of divergence or convergence of orbits in the phase space that start close to each other. Mathematically, LEs are eigenvalues of the state transition matrix that determine evolution of an infinitesimal perturbation in the dynamical system. Hence, they can be treated as a measure of the sensitivity to initial conditions in the phase space.

For preliminary assessment of the system dynamics, it is enough to know the largest Lyapunov exponent. Positive value of the largest LE indicates high sensitivity to initial conditions of the system and is a sign of an irregular response, i.e. chaotic or even hyperchaotic. The largest LE equal to zero means regular, periodic or quasi-periodic system dynamics. If all the LEs are negative, then the limit set is a stable fixed point. However, for more detailed and accurate analysis of the system, the knowledge of the complete spectrum of LEs is essential. For any dynamical system, such spectrum is a set of real numbers sorted in the non-increasing order. Number of LEs is equal to the dimension of the phase space. Knowing all the LE values in the spectrum enables to estimate the fractal dimension of the system attractor (according to the Kaplan-Yorke formula [4]), determine the dimension of the system quasiperiodicity (defined by the number of LEs of zero value) or the scale of hyperchaos (defined by number of positive LEs). On the other hand, Lyapunov exponents also provide a criterion for global stability of the system [2]. From properties of the LEs one can conclude that the sum of all of them is equal to the divergence of the phase flow [5]. If the sum of all components in the spectrum is negative, then the dynamical system under consideration is dissipative and its attractor is globally stable. This sum is equal to zero for any conservative system. In the case of positive sum of all the LEs, an exponential divergence of the phase flow is observed, so the system is globally unstable.

The analytical determination of Lyapunov exponents is possible only in the considerations of simple linear dynamical systems. Owing to this fact, numerical methods are used to calculate LEs in most cases. The first numerical characteristics of chaotic behaviour of the dynamical system, which presented a divergence of neighbouring trajectories, was conducted by Henon and Heiles [6]. An efficient algorithm for calculating the complete spectrum of the Lyapunov exponents based on the Oseledec theorem was independently formulated by both Benettin et al. [7] and Shimada, Nagashima [8]. Then, it was improved by Benettin et al. [9-10], Wolf [11], Parker, Chua [12] and Nusse, Yorke [13]. Recently, another method for the LE spectrum calculation, based

\footnotetext{
* Corresponding author: Andrzej.Stefanski@p.lodz.pl
} 
on the scalar product of vectors, was proposed [14-20]. These techniques allow to calculate the complete spectrum of the LEs. They can be successfully used for many dynamical systems, both autonomous and nonautonomous. But the range of their use is limited by the condition of continuity of the system equations in the analysed time interval. Discontinuity in equations usually cause significant numerical problems, because derivative of the trajectory with respect to the initial conditions (Jacobi matrix) becomes ill conditioned or cannot be calculated at all. Therefore, when typical methods are applied, error of LEs estimation becomes very large or calculation of the LEs is not possible.

In the real world there are many dynamical systems with discontinuities. Among them mechanical systems with impacts, dry friction or piecewise linear stiffness characteristics are especially important. Two decades ago, a method of the LEs calculation based on the Oseledec theorem adapted to dynamical systems with discontinuities was proposed [21]. In order to apply this method, linearization of the equations of motion must be accompanied by a clear statement of the transition conditions while the trajectory is passing through the discontinuity. Another class of methods for the LEs calculation employ reduction of the dynamics of the phase flow in the k-dimensional phase space to a discrete map of a lower dimension, such as a Poincaré map [12], an impact map [22], a local map [23] or a transcendental map [24]. Then, the LEs of such mapping are determined using classical approaches mentioned above. First examples of such an approach can be found in the works by Hilborn [25] and Oestreich [26-27]. The main problem in application of these algorithms is determination of the Jacobi matrix of the mapping, where consecutive iterations are not explicitly defined by a known difference equation, but they are reconstructed from the flow. The example of such a map-based algorithm is the method by Galvanetto [28], who applied implicitly defined maps for calculation of the two largest LEs of the 2-DoF stick-slip system. Other interesting application of such approach is the algorithm for impact oscillators formulated by Souza \& Caldas [24], which exploits the so-called transcendental maps that take into account the solution of integrable differential equations between impacts, supplemented by transition conditions at the instants of the impact. On the other hand, Jin et al. [23] focused on the local mappings of non-smooth systems and found a general calculation method of the spectrum of LEs for k-dimensional non-smooth dynamical systems, which could be generally applied to the systems with rigid or flexible constraints. The largest LE can be estimated also from a one-dimensional mapping of the dynamics of the system - the so-called H-map, without defining the Jacobi matrix [26]. The idea of such an attempt is calculation of the value of the largest LE on the basis of the averaged value of the slope (directional coefficient) of a line tangent do the H-map in the point of its contact with the phase plane trajectory [29]. The next practical method for estimation of the largest LE, elaborated by authors of this paper, is based on the synchronization phenomenon. Previously, we successfully employed this approach for discontinuous oscillators with impacts and friction [30-31], discretetime maps [32] and systems with time delay [33].

Other technique, which can be also applied in the case of systems with discontinuities, is an algorithm of estimation of the LEs from the scalar time signal. This method is based on a procedure of reconstruction of the system attractor from the time dependence of one of the coordinates that was introduced by Takens [34]. The first numerical algorithm for estimation of the largest LE, derived from it, was formulated by Wolf [35]. In the following years, this algorithm has been improved by other researchers in order to estimate the entire spectrum of the LEs and to recognize the so-called spurious Lyapunov exponents [36].

In this paper we demonstrate a method for calculating the spectrum of LEs which is based on the idea of estimation of the Jacobian matrix using small perturbations of the initial orthogonal vectors. This technique is especially useful for non-smooth oscillators, because such estimation procedure allows us to avoid direct calculation of the Jacobi matrix. We have successfully applied this procedure in order to test the hyperbolic properties of the attractor of stick-slip oscillator [37]. Recently, an analogous approach of determining the LEs has been presented by Soriano et al. [38]. They named it cloned dynamics approach. We have developed and applied this technique to a typical mechanical system with discontinuity: the impact oscillator.

The paper is organized as follows. The section 2 contains a detailed description of the applied method of the LEs spectrum calculation. In the section 3 examples of the method application are demonstrated. Finally, in the section 4 , a discussion of our results and conclusions are presented.

\section{The method}

The method under consideration is based on the classical algorithm by Parker \& Chua [12]. However, calculation of linearized equations is replaced with the estimation of Jacobi matrices using small orthogonal perturbation vectors. Scheme of the Jacobi matrix estimation method is presented in the Fig. 1.

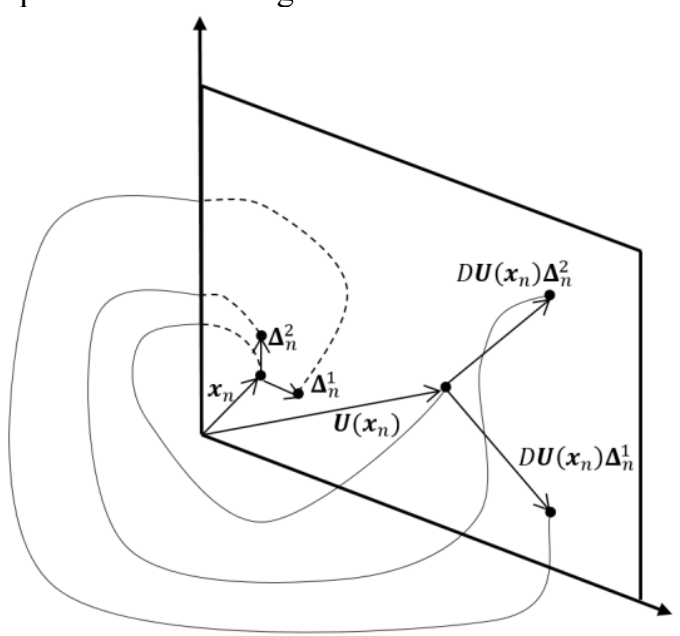

Fig. 1. Scheme of the Jacobi matrix estimation method 
The idea of the estimating procedure is as follows. For any $\mathrm{N}$-dimensional time-continuous system:

$$
\dot{\mathbf{y}}=\mathbf{F}(\mathbf{y})
$$

where $\mathbf{y}=\left[y_{1}, y_{2}, \ldots, y_{N}\right]^{\mathrm{T}} \in R^{N}$ and $\mathbf{F}=\left[f_{1}, f_{2}, \ldots, f_{N}\right]^{\mathrm{T}}$, a $(N-1)$-dimensional map (e.g. Poincaré cross-section) can be defined in the following general form:

$$
\mathbf{x}_{n+1}=\mathbf{U}\left(\mathbf{x}_{n}\right)
$$

where $\mathbf{x}=\left[x_{1}, x_{2}, \ldots, x_{N-1}\right]^{\mathrm{T}} \in R^{N-1}$. For the discrete map presented in the Eq. (2), direct calculation of Jacobi matrices is impossible, because the right side of the Eq. (2) is not known. In this case, each iteration of the map is reconstructed numerically from the solution of the investigated system, described by the Eq. (1). Therefore, the Jacobi matrix of the map (2) cannot be determined analytically. However, an approximate value of the Jacobi matrix can be evaluated by means of the method of small perturbations vectors. This idea works as follows. After introducing the perturbation vector $\Delta n=$ $\left[\delta_{1}, \delta_{2}, \ldots, \delta_{N-1}\right]^{\mathrm{T}}$, where $\delta_{i}(i=1,2, \ldots N-1)$ is a small magnitude value, into the Eq. (2), we have

$$
\mathbf{x}_{n+1}+\Delta_{n+1}=\mathbf{U}\left(\mathbf{x}_{n}+\Delta_{n}\right) \approx D \mathbf{U}\left(\mathbf{x}_{n}\right) \Delta_{n}+\mathbf{U}\left(\mathbf{x}_{n}\right)
$$

where $D \mathbf{U}\left(\mathbf{x}_{n}\right)$ is a Jacobi matrix composed of $(N-1)$ column vectors $D \mathbf{U}_{i}\left(\mathbf{x}_{n}\right)=\left[\partial_{i}\left(x_{1}, \ldots, x_{N-1}\right) / \partial x_{1}, \ldots\right.$, $\left.\partial f_{i}\left(x_{1}, \ldots, x_{N-1}\right) / \partial x_{N-1}\right]^{\mathrm{T}}$, i.e.:

$$
D \mathbf{U}\left(\mathbf{x}_{n}\right)=\left[D \mathbf{u}_{1}\left(\mathbf{x}_{n}\right), D \mathbf{u}_{2}\left(\mathbf{x}_{n}\right), \ldots, D \mathbf{u}_{N-1}\left(\mathbf{x}_{n}\right)\right](4)
$$

From Eq.(3) the Jacobi matrix can be approximated from the formula:

$$
D \mathbf{U}\left(\mathbf{x}_{n}\right) \boldsymbol{\Delta}_{n} \approx \mathbf{U}\left(\mathbf{x}_{n}+\boldsymbol{\Delta}_{n}\right)-\mathbf{U}\left(\mathbf{x}_{n}\right)
$$

Consequently, each column vector can be estimated as:

$$
D \mathbf{u}_{i}\left(\mathbf{x}_{n}\right) \approx\left[\mathbf{U}\left(\mathbf{x}_{n}+\boldsymbol{\Delta}_{n}^{i}\right)-\mathbf{U}\left(\mathbf{x}_{n}\right)\right] / \delta
$$

where $\quad \Delta_{n}{ }^{1}=\left[\begin{array}{lll}\delta, & 0, \ldots, & 0\end{array}\right]^{\mathrm{T}}, \quad \boldsymbol{\Delta}^{2}=\left[\begin{array}{lll}0, & \delta, \ldots, & 0\end{array}\right]^{\mathrm{T}} \ldots$, $\Delta n^{N-1}=[0,0, \ldots, \delta]^{\mathrm{T}}$ are orthogonal vectors of small perturbation $\delta$. Columns of the Jacobi matrix of the map $D \mathbf{u}_{i}\left(\mathbf{x}_{n}\right)$ can be estimated numerically from the solution of the system defined by the Eq. (1). Procedure is as follows. For each iteration of the map, trajectory has to be calculated once for the reference path, starting from the original initial conditions $\mathbf{x}_{n}$, and $N-1$ times for the initial conditions disturbed by each perturbation vector: $\mathbf{x}_{n}+\Delta n^{i}$. In such manner, after the iteration of the map, the reference point $\mathbf{x}_{n+l}=\mathbf{U}\left(\mathbf{x}_{n}\right)$ and $N$-1 perturbed points $\mathbf{U}\left(\mathbf{x}_{n}+\boldsymbol{\Delta}_{n}{ }^{i}\right) \approx \approx \mathbf{x}_{n+l}+D \mathbf{U}\left(\mathbf{x}_{n}\right) \Delta_{n+1}{ }^{i}$ are obtained (see the Fig. 1). Then, columns of the Jacobi matrix described in the Eq. (4) can be obtained from the Eq. (6). Obviously, this way of Jacobian matrix estimation can be also applied for the maps given by known difference equations.
Let's follow how this procedure works on the example of classical Henon map, i.e.:

$$
\mathbf{U}\left(\mathbf{x}_{n}\right)=\left\{\begin{array}{c}
x_{n+1}=1-a x_{n}^{2}+y_{n} \\
y_{n+1}=b x_{n}
\end{array}\right.
$$

with the Jacobi matrix :

$$
D \mathbf{U}\left(\mathbf{x}_{n}\right)=\left[\begin{array}{cc}
-2 a x_{n} & 1 \\
b & 0
\end{array}\right]
$$

Orthogonal perturbation vectors are $\Delta_{x}=\left[\begin{array}{ll}\delta, & 0\end{array}\right]^{\mathrm{T}}$ and $\Delta_{y}=[0, \delta]^{\mathrm{T}}\left(\delta_{x}=\delta_{x}=\delta\right)$. Substituting Henon map into the Eq.(6), we obtain following column vectors:

$$
\begin{aligned}
& D \mathbf{u}_{x}\left(\mathbf{x}_{n}\right)=\left[\mathbf{U}\left(\mathbf{x}_{n}+\boldsymbol{\Delta}_{x}\right)-\mathbf{U}\left(\mathbf{x}_{n}\right)\right] / \delta= \\
& =\left[\begin{array}{c}
\frac{1-a\left(x_{n}+\delta\right)^{2}+y_{n}-\left(1-a x_{n}^{2}+y_{n}\right)}{\delta} \\
\frac{b\left(x_{n}+\delta\right)-b x_{n}}{\delta}
\end{array}\right]=\left[\begin{array}{c}
-2 a\left(x_{n}+\delta\right) \\
b
\end{array}\right](9)
\end{aligned}
$$

Finally, in accordance with the Eq.(4), estimated Jacobi matrix of the Henon map is obtained in the following form:

$$
D \mathbf{U}\left(\mathbf{x}_{n}\right)=\left[\begin{array}{ll}
D \mathbf{u}_{x}\left(\mathbf{x}_{n}\right) & D \mathbf{u}_{y}\left(\mathbf{x}_{n}\right)
\end{array}\right]=\left[\begin{array}{cc}
-2 a\left(x_{n}+\delta\right) & 1 \\
b & 0
\end{array}\right](10)
$$

The example above implies that the precision of the Jacobi matrix estimation depends on the magnitude of the small parameter $\delta$. Note that, in general, this procedure requires high accuracy of the trajectory simulation, especially in the vicinity of the discontinuity. Then, such numerically generated Jacobi matrix can be implemented in a classical algorithm for calculating Lyapunov exponents spectrum, including the GrammSchmidt orthonormalization [12].

\section{Numerical example}

In this paper, the method presented in the previous section has been applied to a simple mechanical system with impacts. Scheme of the system is presented in the Fig. 2. The system is a typical linear mass-spring-damper system. However, motion of the mass is constrained by the wall placed in the position $X_{w}$. The equation of motion of the system is as follows:

$$
m \ddot{X}=-c \dot{X}-k X+F \cos (\Omega t)
$$

where $X$ is the coordinate of the oscillator, $m$ is the mass of the oscillator, $k$ is the spring stiffness, $c$ is the damping coefficient, $F$ is the amplitude of forcing, $\Omega$ is the angular frequency of forcing and $X_{w}$ is the position of the wall. Obviously, this equation is not correct when a contact with the wall occurs. If $t_{c}$ is the moment of time in which collision with the wall takes place, then transition of the oscillator's velocity is defined by the following equation: 


$$
\dot{X}\left(t_{c}^{+}\right)=-e \dot{X}\left(t_{c}^{-}\right)
$$

where $e$ is the coefficient of restitution (CoR).
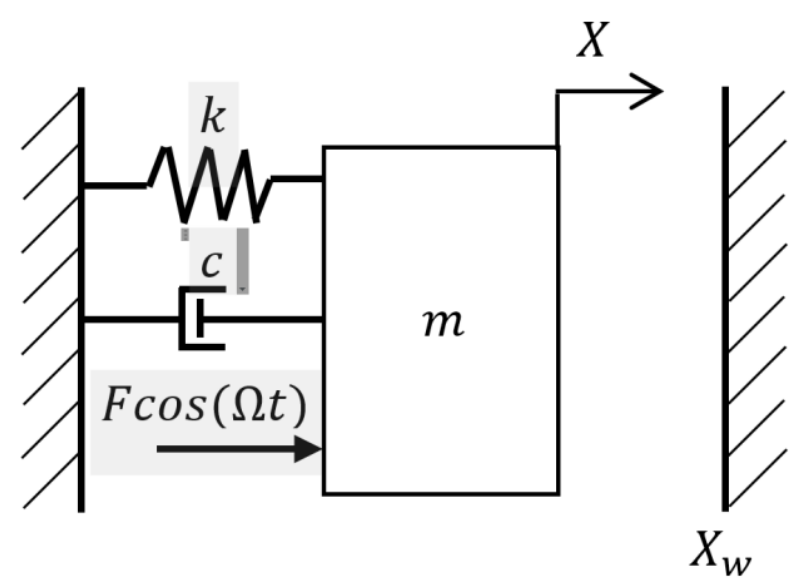

Fig. 2. Scheme of the analysed system with impacts

By simple rearrangements, Eq. (11-12) can be transformed to the form:

$$
\begin{gathered}
X^{\prime \prime}=-2 \zeta X^{\prime}-X+f \cos (\eta \tau) \\
X^{\prime}\left(\tau_{c}^{+}\right)=-e X^{\prime}\left(\tau_{c}^{-}\right)
\end{gathered}
$$

where $\zeta$ is the damping ratio, $\omega^{2}=k / m$ is the squared natural frequency of the system, $f=F /\left(m \omega^{2}\right), \tau=\omega t$ is the dimensionless time, $\eta=\Omega / \omega$ is the dimensionless frequency of forcing and $\tau_{c}$ is the dimensionless time of collision. Equations (13-14) have been simulated numerically. The values of parameters were chosen as follows: $\zeta=0.05, f=1.0, X_{w}=2.0, \quad e=1.0$ and the dimensionless forcing frequency $\eta$ was used as the control parameter. To simulate the system a simulation program has been written in $\mathrm{C}++$ by means of CodeBlocks environment. Throughout the simulation, Runge-Kutta method of the fourth order has been used to integrate the equation (13). The selected step length was dependent on the dimensionless frequency in such a way, that 1800 integration steps were performed for each forcing period. In order to detect the collision time $\tau_{c}$ with a proper accuracy, the linear interpolation method has been used when the coordinate $X$ approached $X_{w}$. The spectrum of Lyapunov exponents has been calculated by means of the method described in the previous section. The obtained bifurcations diagrams and Lyapunov exponents graphs are presented in the Fig. 3.

\section{Observations and conclusions}

It can be noticed that the bifurcation diagram presented in the Fig. 3 is consistent with the Lyapunov exponents graph. Moreover, it can be shown that the divergence of the system (13-14) is equal to $2 \zeta=-0.1$. As one can notice in the graph, the sum of both Lyapunov exponents from the spectrum is also equal to
-0.1 independently of the value of the control parameter $\eta$, which confirms validity of calculations.

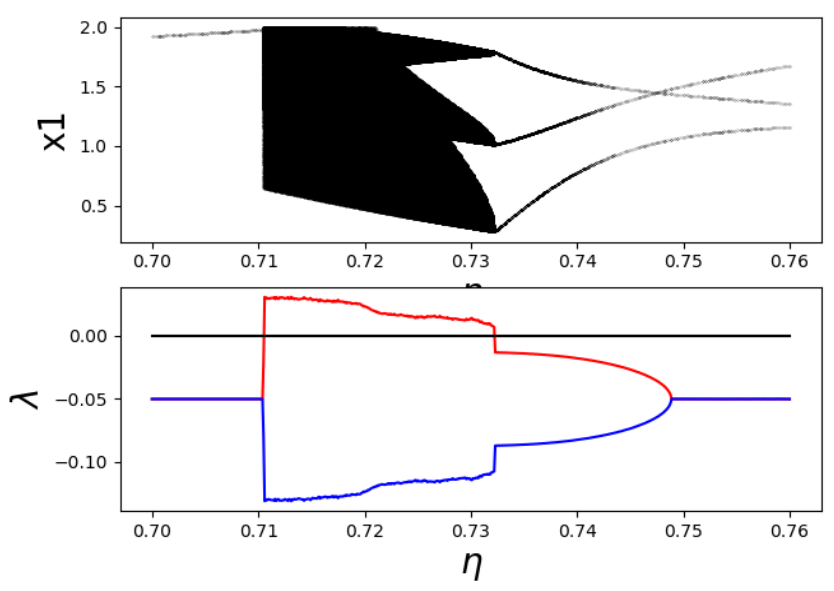

Fig. 3. Bifurcation diagram and Lyapunov exponents graphs of the system defined by the Eq. (21-22)

This paper provides an introduction to the new method of Lyapunov exponents spectrum estimation for non continuous dynamical systems. Foundations of the method have been explained and detailed description of the algorithm has been given. The numerical example proves validity and efficiency of the method. It has been shown that this method of LEs spectrum estimation can be successfully applied to mechanical systems with discontinuities. In the future, authors plan to apply the method to other kinds of non continuous mechanical systems, for example as stick-slip oscillator or a network of such oscillators.

This study has been supported by Polish Ministry of Science and Higher Education under the program "Diamond Grant", project no. D/2013 019743 .

This study has been supported by Polish National Centre of Science (NCN) under the program MAESTRO: "Multi-scale modelling of hysteretic and synchronous effects in dry friction process", project no. 2012/06/A/ST8/00356.

\section{References}

1. A. M. Lyapunov: Probleme General de la Stabilité du Mouvment. Annales Mathematical Study, 17, Princeton University Press, Princeton, New Jersey (1947)

2. G. D. Birkhoff: Dynamical Systems, AMS Colloquium Publications, Providence, (1927)

3. V. I. Oseledec: A multiplicative ergodic theorem: Lyapunov characteristic numbers for dynamical systems. Trans. Mosc. Math. Soc. 19, 197-231 (1968)

4. J. L. Kaplan, J. A. Yorke: Chaotic behavior of multidimensional difference equations, in Lecture Notes in Mathematics, vol. 730, edited by H. O. Peitgen and H. O. Walther, pp. 228 -- 237, Springer, Berlin (1978) 
5. V. C. Anishchenko: Complex oscillations in simple systems, 312, Nauka Publisher, Moscow (1990)

6. M. Henon, C. Heiles: The applicability of the third integral of the motion: some numerical results, Astron. J. 69, 77 (1964)

7. G. Benettin, L. Galgani, J.M. Strelcyn: Kolmogorov entropy and numerical experiment, Phys. Rev. A 14, $2338-2345$ (1976)

8. I. Shimada, T. Nagashima: A numerical approach to ergodic problem of dissipative dynamical systems, Prog. Theor. Phys. 61(6), 1605-1616 (1979)

9. G. Benettin, L. Galgani, A. Giorgilli, J.M. Strelcyn: Lyapunov exponents for smooth dynamical systems and Hamiltonian systems; a method for computing all of them, part I: theory, Meccanica 15, 9-20 (1980)

10. G. Benettin, L. Galgani, A. Giorgilli, J.M. Strelcyn: Lyapunov exponents for smooth dynamical systems and Hamiltonian systems; a method for computing all of them, part II: numerical application. Meccanica 15, 2130 (1980)

11. A. Wolf: Quantifying chaos with Lyapunov exponents, Chaos, Manchester University Press, Manchester, 273-290 (1986)

12. T.S. Parker, L.O. Chua: Practical Numerical Algorithms for Chaotic Systems, Springer-Verlag, Berlin (1989)

13. H. Nusse, J. Yorke: Dynamics: Numerical Explorations, Springer-Verlag (1994)

14. A. Dąbrowski: Estimation of the largest Lyapunov exponent from the perturbation vector and its derivative dot product, Nonlinear Dynamics 67(1), 283-291 (2012)

15. M. Balcerzak, A. Dąbrowski, D. Pikunov: The fastest, simplified method of Lyapunov exponents spectrum estimation for continuous dynamical systems, Submitted to Nonlinear Dynamics

16. A. Dąbrowski: The largest transversal Lyapunov exponent and master stability function from the perturbation vector and its derivative dot product (TLEVDP), Nonlinear Dynamics 69(3), 1225-1235 (2012)

17. M. Balcerzak, A. Dąbrowski, T. Kapitaniak, A. Jach: Optimization of the control system parameters with use of the new simple method of the largest Lyapunov exponent estimation, Mechanics and Mechanical Engineering 17(3), 225-239 (2013)

18. K. Pijanowski, A. Dąbrowski, M. Balcerzak: New method of multidimensional control simplification and control system optimization, Mechanics and Mechanical Engineering 19(2), 127-139 (2015)

19. A. Dąbrowski: Estimation of the the Largest Lyapunov exponent-like (LLEL) stability measure parameter from the perturbation vector and its derivative dot product (part 2) experiment simulation, Nonlinear Dynamics 78(3), 1601-1608 (2014)

20. M. Balcerzak, D. Pikunov: The fastest, simplified method of estimation of the Largest Lyapunov exponent for continuous dynamical systems with time delay, Mechanics and Mechanical Engineering (accepted)

21. P. Müller: Calculation of Lyapunov exponents for dynamical systems with discontinuities, Chaos, Solitons Fractals 5(9), 1671-1681 (1995)

22. A. Stefański, T. Kapitaniak: Estimation of the dominant Lyapunov exponent of non-smooth systems on the basis of maps synchronization, Chaos, Solitons \& Fractals, 15(2), pp. 233-244 (2003)

23. L. Jin, Q.-S. Lu, E.H. Twizell: A method for calculating the spectrum of Lyapunov exponents by local maps in non-smooth impact-vibrating systems, Journal of Sound and Vibration, 298(4-5), pp. 1019-1033 (2006)

24. S. De Souza, I. Caldas: Controlling chaotic orbits in mechanical systems with impacts, Chaos, Solitons \& Fractals, 19, 171-178 (2004)

25. R. C. Hilborn: Chaos and Nonlinear Dynamics: An Introduction for Scientists and Engineers, Oxford University Press (1994)

26. M. Oestreich, N. Hinrichs, K. Pop : Bifurcation and stability analysis for a non-smooth frictional oscillator, Arch. Appl Mech. 66, 301-314 (1996)

27. M. Oestreich: Untersuchung von Schwingern mit nichtglatten Kennlinien, Fortsrchritt-Berichte VDI, Reiche 11: Schwingungstechnik, Nr. 258 (in German) (1998)

28. U. Galvanetto: Numerical computation of Lyapunov exponents in discontinuous maps implicitly defined, Computer Physics Communications, 131(1-2), 1-9 (2000)

29. N. Hinrichs, M. Oestreich, K. Popp: Dynamics of oscillators with impact and friction, Chaos, Solitons \& Fractals, 4(8), 535-58 (1997)

30. A. Stefański, T. Kapitaniak: Using chaos synchronization to estimate the largest Lyapunov exponent of non-smooth systems, Discrete Dyn. Nat. Soc. 4, 207-215 (2000) 
31. A. Stefański: Estimation of the largest Lyapunov exponent in systems with impacts, Chaos, Solitons Fractals 11(15), 2443-2451 (2000)

32. A. Stefański, T. Kapitaniak: Estimation of the dominant Lyapunov exponent of non-smooth systems on the basis of maps synchronization, Chaos, Solitons Fractals 15, 233-244 (2003b)

33. A. Stefański, A. Dąbrowski, T. Kapitaniak: Evaluation of the largest Lyapunov exponent in dynamical systems with time delay, Chaos Solitons Fractals 23, 1651-1659 (2005)

34. F. Takens: Detecting strange attractors in turbulence, Lecture Notes in Mathematics, 898(1), 366$381(1981)$

35. A. Wolf, J.B. Swift, H.L. Swinney, J.A. Vastano: Determining Lyapunov exponents from a time series, Physica D: Nonlinear Phenomena, 16(3), 285-317 (1985)

36. J.A. Tempkin, J.A. Yorke: Spurious Lyapunov exponents computed from data, SIAM Journal on Applied Dynamical Systems, 6(2), 457-474 (2007)

37. A. Stefański, J. Wojewoda, A. Chudzik, T. Kapitaniak: Hyperbolic-like properties of Popp's attractor, International Journal of Bifurcation and Chaos, 22(08), 1250187 (2012)

38. D.C. Soriano, F.I. Fazanaro, R. Suyama, J.R. de Oliveira, R. Attux, M.K. Madrid: A method for Lyapunov spectrum estimation using cloned dynamics and its application to the discontinuously-excited FitzHugh-Nagumo model, Nonlinear Dynamics, 67(1), 413-424 (2012) 